Department of Paediatric Cardiology

Sakakibara Heart Institute, Tokyo,

Japan

K Suzuki

Y Murakami

S Mimori

Correspondence to:

Dr K Suzuki, Department of

Paediatric Cardiology, Sakakibara Heart Institute,

5-4 Yoyogi 2-chome

Shibuya-ku Tokyo 151, Japan.

\section{Multiple arterial stenoses of unknown aetiology in the coronary, cerebral, and brachial arteries}

\author{
Kiyoshi Suzuki, Yasuo Murakami, Shigekazu Mimori
}

rounded by abundant fibrous tissue. Percutaneous transluminal angioplasty of the right coronary stenoses led to complete occlusion.

(Br Heart $\mathcal{F}$ 1993;70:386-390)

In 1991 we described a girl with multiple coronary stenoses of unknown aetiology ${ }^{1}$ and we also reported on an adolescent boy with silent coronary ischaemia. ${ }^{2}$ We now report another adolescent girl who had both silent myocardial ischaemia with multiple coronary stenoses and multiple lesions in the cerebral
।

aVF

$7 \mathrm{mnth}$
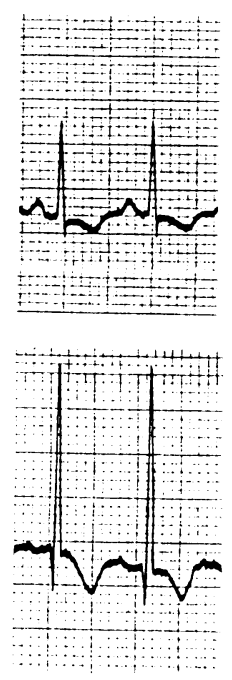

V1
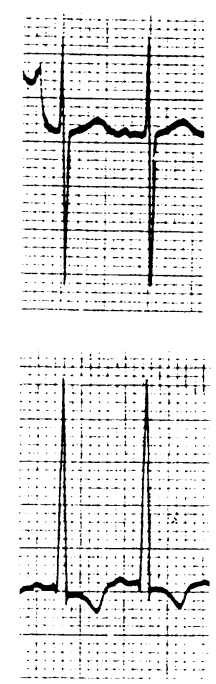
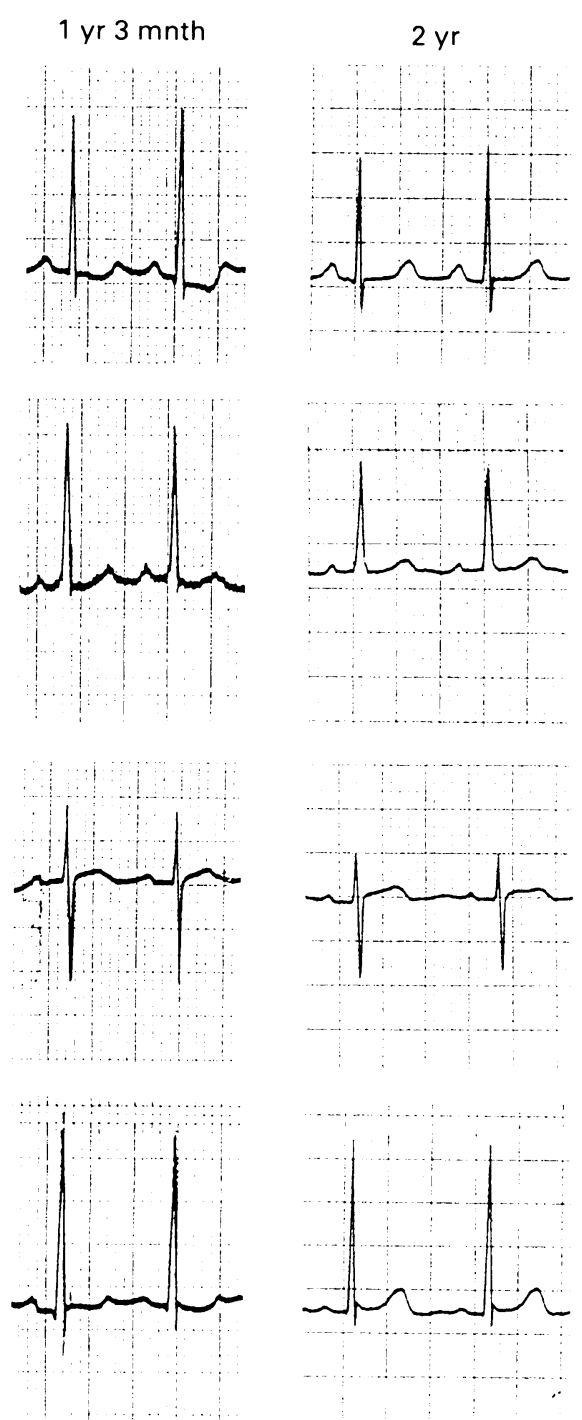

$12 \mathrm{yr}$
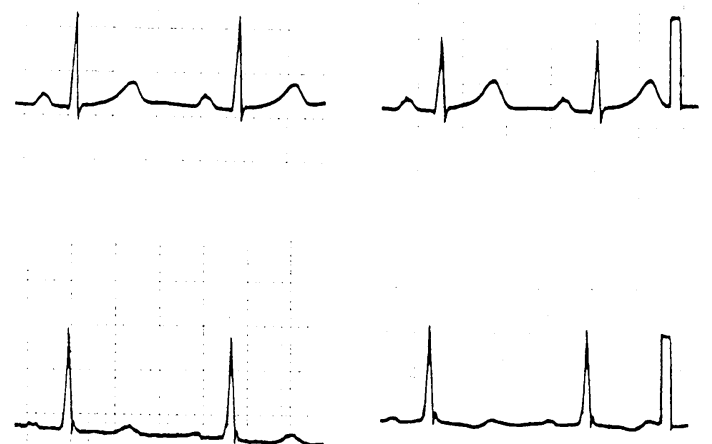<smiles>CCCCCCCCCCCC</smiles><smiles>C=CCCCCCC(C)C</smiles>

Figure 1 Electrocardiographic changes, electrocardiogram recorded when the patient was seven months old, showing left ventricular hypertrophy with ST segment depression and inverted $T$ wave in leads $I, a V F$, and V5. These patterns gradually improved. When she was 12 she had an almost normal electrocardiogram, even after complete occlusion of the right coronary artery (RCA). 


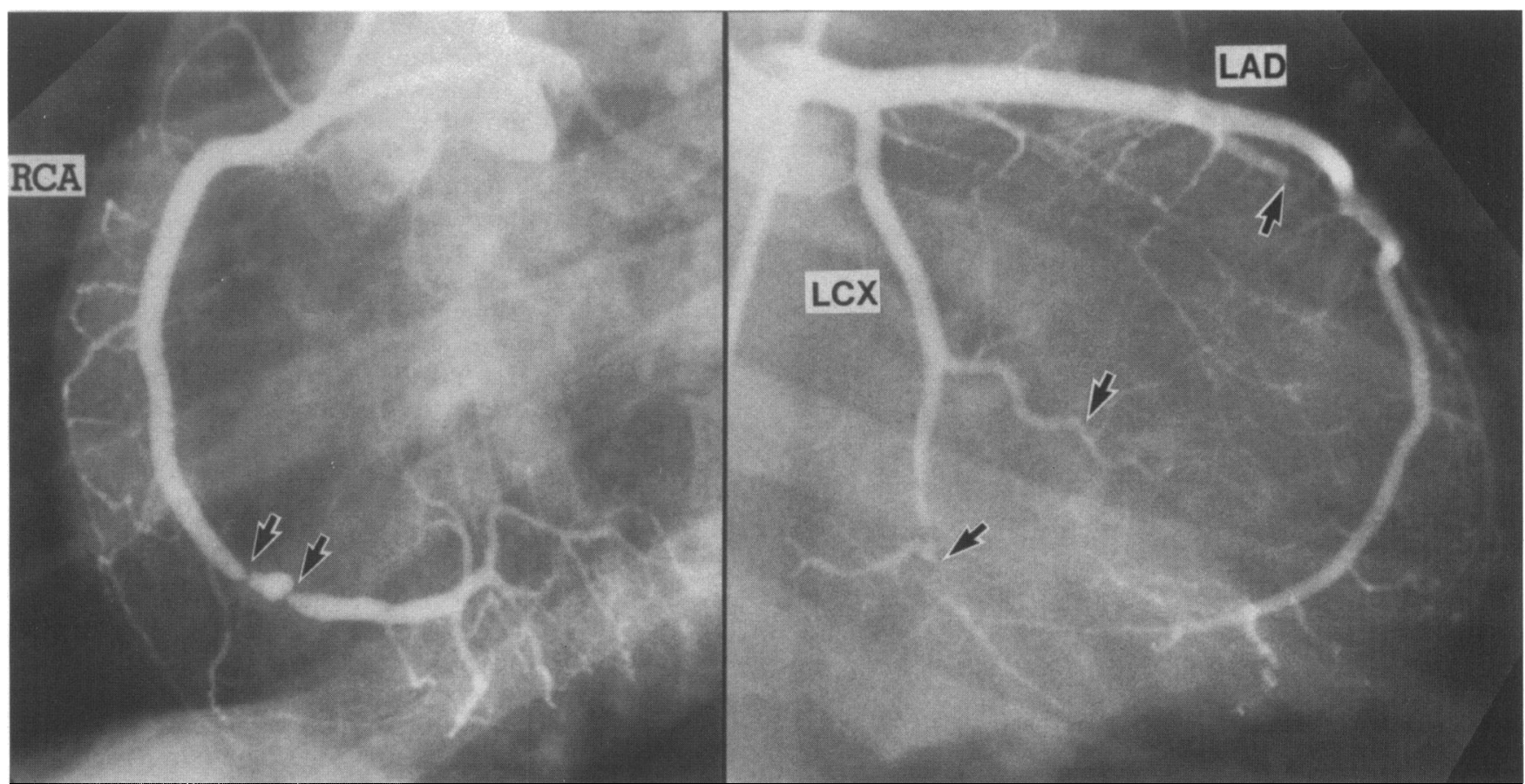

Figure 2 Multiple coronary stenoses (arrows) in both coronary arteries. RCA; right coronary artery; LAD; left anterior descending artery; LCX; left circumflex artery.

arteries and the left brachial artery. Endocardial fibroelastosis was diagnosed in early infancy and since then her cardiac function has gradually improved. This unusual patient had no history of any disorder that could have caused generalised arterial involvement in childhood.

\section{Case report}

Four months before cardiomegaly was found on the chest $x$ ray (CTR 74\%) and left ventricular hypertrophy on the electrocardiogram (fig 1) (at the age of seven months) this girl had suffered from tachypnoea, wheezing, and poor sucking. The left ventriculogram showed a diminished left ventricular ejection fraction (EF 23\%) without any cardiac anomalies. No occlusive lesion was noted in the main coronary arteries. Endocardial fibroelastosis was diagnosed. Her symptoms gradually improved when she was treated with digoxin and diuretics.

At the age of two she had headache and nausea caused by hypertension $(178 / 110 \mathrm{~mm}$ $\mathrm{Hg}$ right arm and $160 / 100 \mathrm{~mm} \mathrm{Hg}$ left arm). She was not overweight and renal function was normal. The serum concentration of renin was high $(15.9 \mathrm{ng} / \mathrm{ml}$; normal $10 \cdot 2-2 \cdot 7)$

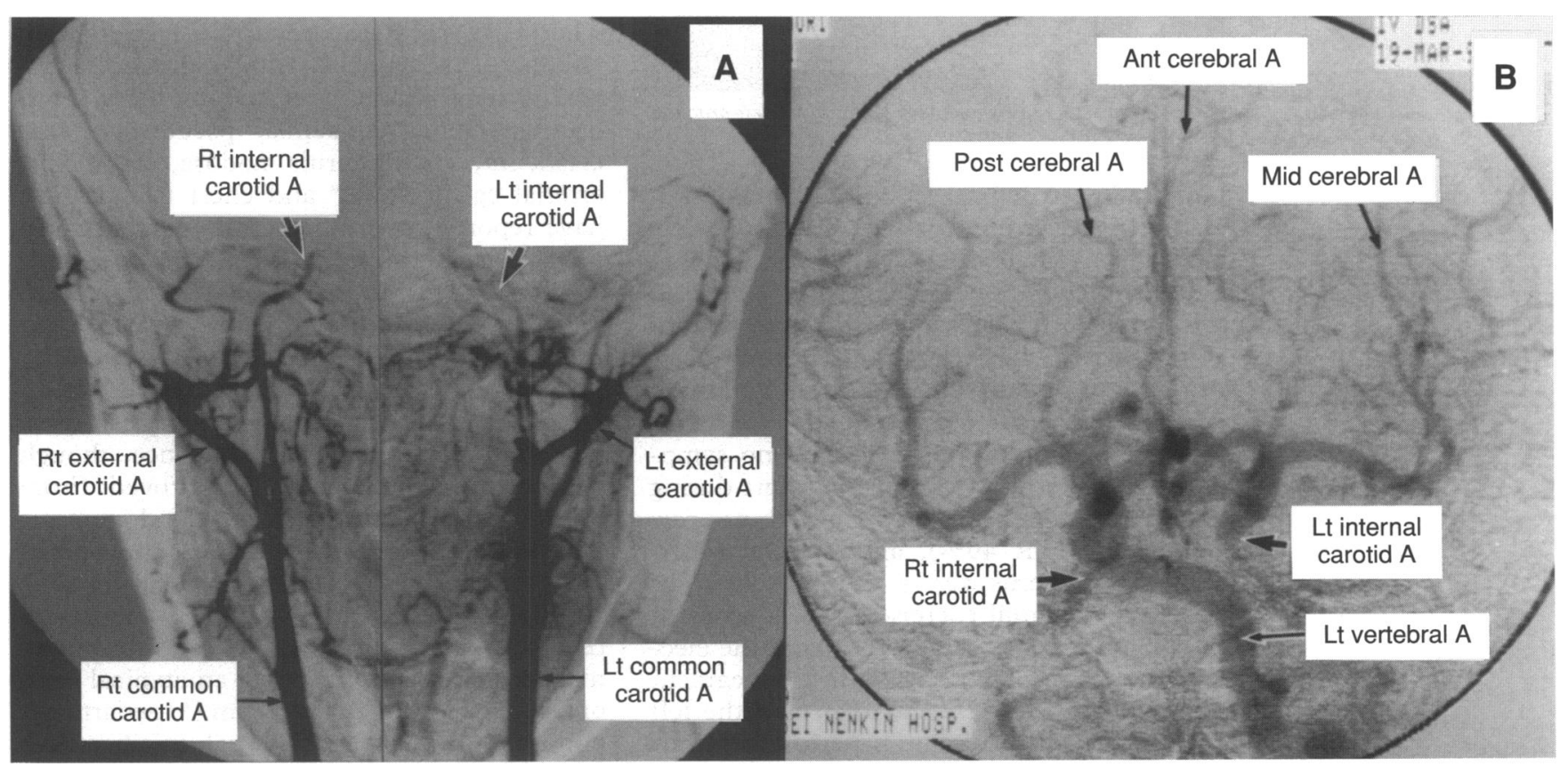

Figure 3 Angiograms of the cerebral arteries. (A) Angiograms of the common carotid arteries showing complete occlusion (large arrows) of both internal carotid arteries. (B) Dilated left vertebral artery supplying blood to the entire brain. Rt, right; Lt, left; Ant, anterior; Mid, middle; Post, posterior; A, artery. 


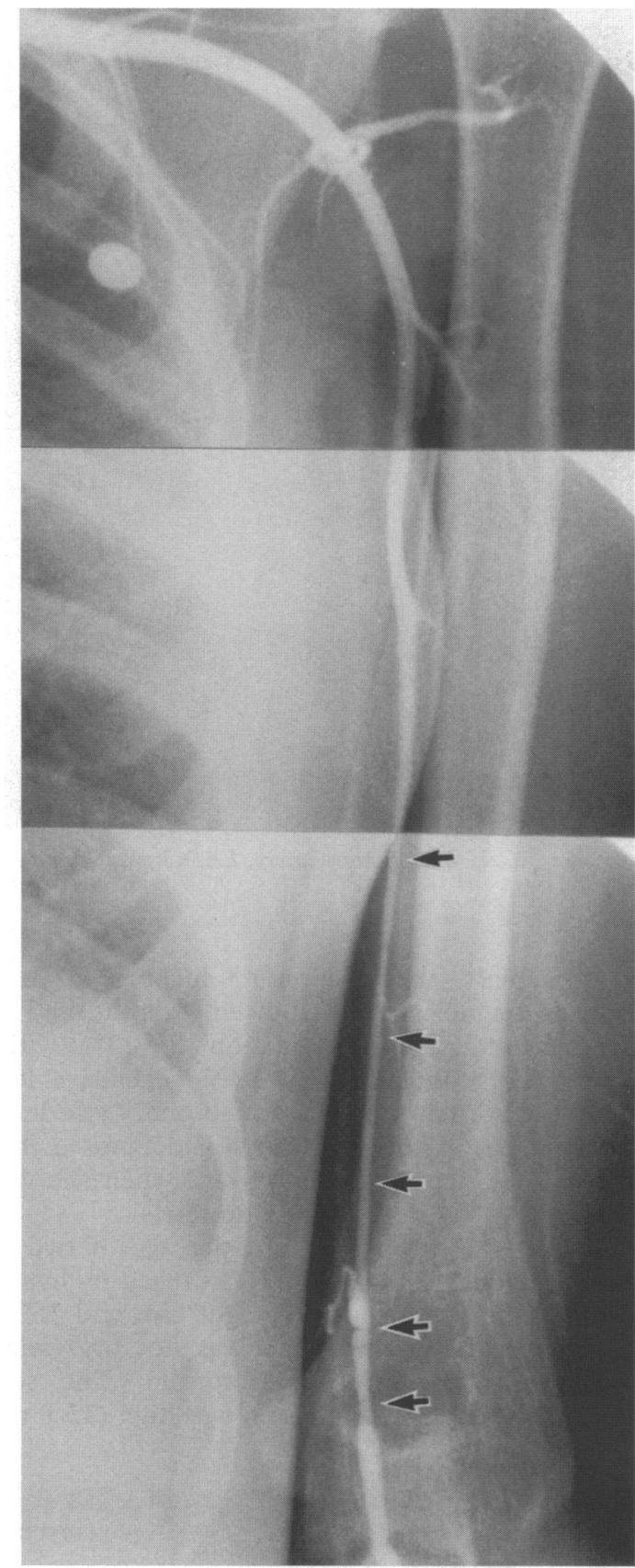

Figure 4 Angiograms showing stenoses of the left brachial artery (arrows).

though the structure of both renal arteries was normal. Cardiomegaly (CTR 65\%), electrocardiographic findings (fig 1), and left ventricular function (by angiography) (EF 50\%) were improved. She was treated with a converting enzyme inhibitor and her blood pressure became normal and her condition remained satisfactory for nine years.

At the age of 11 years she had no symptoms except for mild chest discomfort during exercise. The chest $x$ ray showed no cardiomegaly (CTR 49\%), and the electrocardiographic patterns were within normal limits (fig 1). A treadmill exercise test was stopped because of the chest discomfort, and the electrocardiogram showed 2-3 mm ST segment depression in leads II, III, aVF, and the left precordial leads. A thallium perfusion scintigram also showed ischaemic change in the anterolateral and posterior walls of the left ventricle. Coronary angiography showed mul- tiple stenoses in both coronary arteries (fig 2) but cardiac function was almost normal (EF $60 \%$ ). Moreover, both internal carotid arteries and the right vertebral artery were occluded, and the dilated left vertebral artery supplied blood to the whole brain (fig 3 ). Neurological examination and computed tomography of the brain did not show any abnormalities. There were also stenoses in the long segment of the left brachial artery (fig 4). Blood pressure in the upper arms was $135 / 75$ $\mathrm{mm} \mathrm{Hg}$ and $75 / 58 \mathrm{~mm} \mathrm{Hg}$. There were no stenoses in the abdominal arteries, including the renal arteries; and the serum renin concentration was within the normal range. Biopsy specimens of endomyocardium from the right ventricle showed hypertrophic muscles surrounded by abundant fibrous tissue (fig 5). Her skin and optic fundi were normal.

Anterolateral ischaemia of the left ventricle was caused by multiple stenoses of the small arteries arising from the major left coronary arteries. We performed percutaneous transluminal angioplasty of the right coronary lesion when she was 12 years old. Despite an initial improvement in the stenoses an angiogram 12 days after the procedure showed complete occlusion. The distal part of the right coronary artery was supplied by collaterals from the left coronary artery. Neither her symptoms nor the ischaemic patterns in the electrocardiogram (fig 1) and thallium scintigram have worsened. She was advised not to participate in strenuous sports and was prescribed a $\beta$ blocker and vasodilator. She has remained well for a year.

Blood analyses have never shown any abnormal findings related to carditis or disorders leading to coronary ischaemia. Physical examination of her family members and their histories were normal.

\section{Discussion}

There are several coronary risk factors in childhood, such as Kawasaki disease, ${ }^{3}$ congenital malformations of the coronary arteries, hypercholesterolaemia, ${ }^{4}$ pseudoxanthoma elasticum, ${ }^{56}$ periarteritis nodosa, ${ }^{7}$ idiopathic arterial calcification, ${ }^{8}$ and chest trauma. We have reported two other cases of silent coronary ischaemia of unknown aetiology in adolescents. ${ }^{12}$ The coronary lesion in the present patient resembles that in an earlier case, ${ }^{1}$ but the clinical course was quite different. The patient we report here had had severe heart failure in early infancy; and multiple arterial stenoses were found in adolescence, though her symptoms had gradually improved. From the histological examination of endomyocardial specimens we assume that the most probable cause of her disorder was myocarditis and polyarteritis in early infancy, even though repeated blood analyses showed no abnormalities. She could have had an atypical form of pseudoxanthoma elasticum, ${ }^{56}$ periarteritis nodosa, ${ }^{7}$ or idiopathic arterial calcification ${ }^{8}$ : however, she had no other features of these disorders, such as pigmentation or papules on her skin, angioid streaks on the retinas, or 


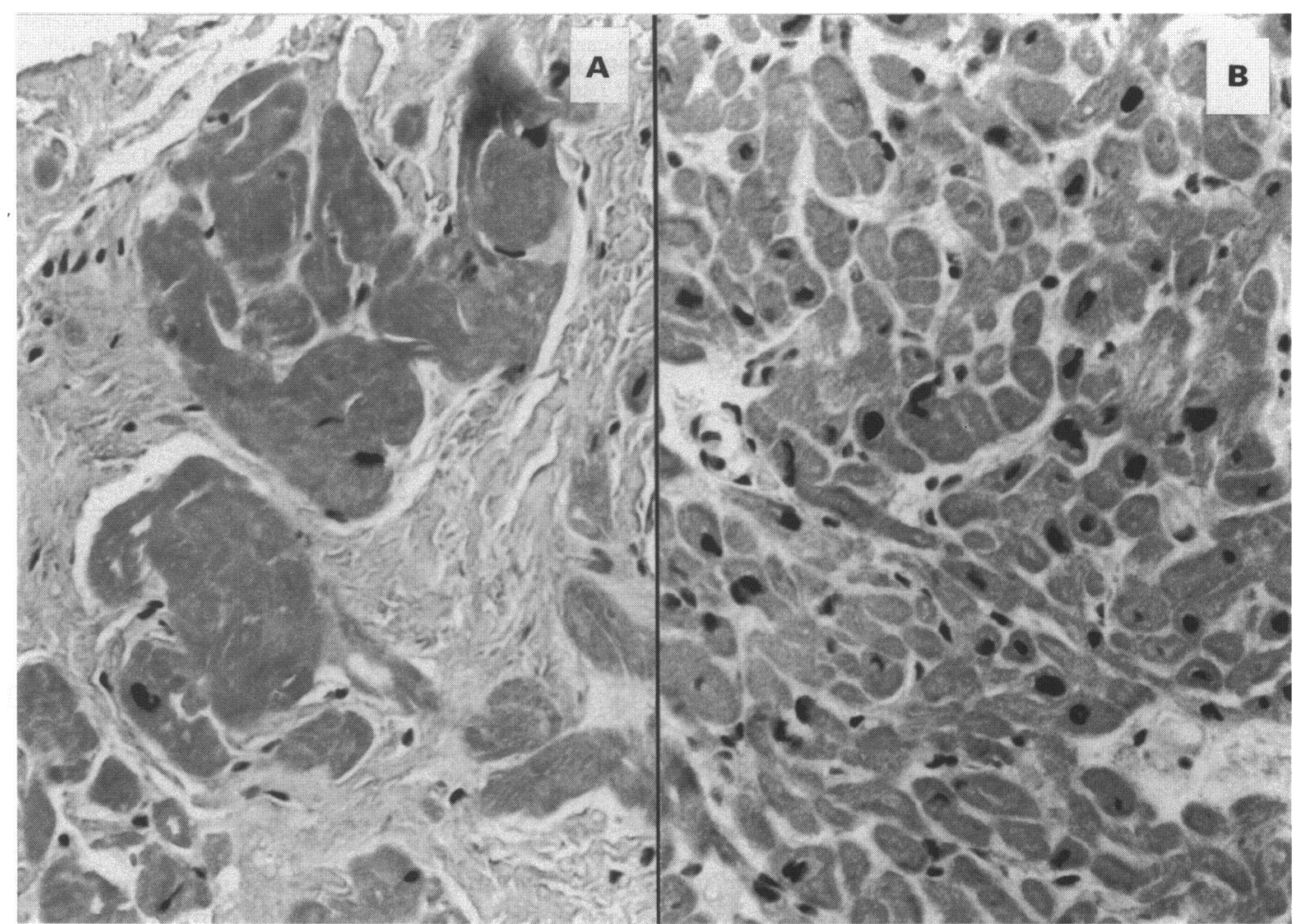

Figure 5 Biopsy specimens from the right ventricle show different histological appearances-from hypertrophic muscle surrounded by abundant fibrous tissue $(A)$ to an almost normal structure $(B)$.

abnormal calcification. Fibromuscular dysplasia can affect the renal, cerebral, and brachial arteries, ${ }^{910}$ however, there are no reports of heart failure in early infancy in this condition.

The reason for her systemic hypertension and high renin concentrations when she was two years old remains unclear. There was no evidence of renal arterial stenosis or renal failure, the major causes of secondary hypertension in infancy. We used a converting enzyme inhibitor, which is one of the most useful drugs for symptomatic hypertension, whatever the serum renin concentration. ${ }^{1112}$ Subsequently she did not have hypertension or any side effect for nine years. She safely stopped taking the drug at the age of 11 years when the serum renin concentration was found to be normal.

Left coronary stenoses, the major cause of her ischaemia, were not a strong indication for angioplasty, because all the lesions were in small arteries, except for the proximal part of the diagonal branch. We predicted that severe symptoms would develop if the right coronary artery became occluded in the presence of broad anterolateral ischaemia. So we chose as the first procedure coronary angioplasty to the right coronary stenoses, despite an absence of severe ischaemia of the inferior myocardium. Fortunately, a good collateral circulation has prevented the development of symptoms after occlusion of the right coronary artery, and we have not done any other intervention for her myocardial ischaemia.

Echigo $^{13}$ and Ino et $a l^{14}$ reported the successful percutaneous transluminal angioplasty of coronary lesions caused by Kawasaki disease. Angioplasty of this patient's coronary lesion failed. If her myocardial ischaemia is not controlled by medical treatment we shall consider bypass surgery to the distal part of the right coronary artery or the diagonal branch. Coronary angioplasty of the diagonal branch may be another choice, but there is some risk of complete occlusion. The best treatment for coronary stenosis in childhood, especially for multiple lesions of unknown aetiology, remains to be determined.

Patients with silent coronary ischaemia can die suddenly during strenuous exercise. All children with cardiomegaly and diminished cardiac function should be carefully observed even if they remain well.

We thank Dr Shin Suzuki, Dr Masateru Oonaka, Dr Kazuyuki Kitamura, and Dr Fred Shane for their help with this paper. This work was supported by a research grant from the Vehicle Racing Commemorative Foundation, Tokyo, Japan.

1 Suzuki K, Murakami Y, Sakai A, Sagara H, Hatai Y, Mori $\mathrm{K}$, et al. Multiple coronary stenoses of unknown etiology. Pediatr Cardiol 1991;12:102-4.

2 Suzuki K, Murakami Y, Mori K, Sakai A, Hatai Y, Sagara $\mathrm{H}$, et al. Silent myocardial ischemia of unknown etiology in childhood. (English abstract) $\mathcal{f} f$ Pediatr Soc in childhood. (Eng

3 Tatara K, Kusakawa $S$, Itoh $K$, Kazuma $N$, Lee $K$ Hashimoto $\mathrm{K}$, et al. Long-term prognosis of Kawasaki disease patients with coronary artery obstruction. Hear Vessels 1989;5:47-51.

4 A joint statement for physicians by the Committee on Atherosclerosis and Hypertension in Childhood of the Council of Cardiovascular Disease in the Young and the Nutrition Committee, American Heart Association Diagnosis and treatment of primary hyperlipidemia in childhood. Circulation 1986;74:1181-8.

5 Schachner I, Young D. Pseudoxanthoma elasticum with severe cardiovascular disease in a child. Am $\mathcal{f} D$ is Child 1974;127:571-5.

6 Nishida $H$, Endo $M$, Koyanagi $H$, Ichihara $T$, Takao A, Maruyama $M$. Coronary artery bypass in a 15 -year-old girl with pseudoxanthoma elasticum. Ann Thorac Surg 1990;49:483-5.

7 Corbeel L, Gewillig M, Baeten E, Van Daele MC, Eggermont $\mathrm{E}$. Carotid and coronary artery involvement in infantile periarteritis nodosa possibly induced by 
Coxsackie B4 infection. Eur $\mathcal{F}$ Pediatr 1987;146:441-2.

8 Van Reempts PJ, Boven KJ, Spitaels SE, Roodhooft AM

Vercruyssen ELJ, Van Acker KJ. Idiopathic arteria calcification of infancy. Calcif Tissue Int 1991;48:1-6.

9 Deal JE, Snell MF, Barratt TM, Dillon MJ. Renovascular disease in childhood. $₹$ Pediatr 1992;121:378-84.

10 Cheu HW, Mills JL. Digital artery embolization as a result of fibromuscular dysplasia of the brachial artery. $f$ Vasc of fibromuscular dys

11 Sinaiko AR, Mirkin BL, Hendrick DA, Green TP, O'Dea RF. Antihypertensive effect and elimination kinetics of captopril in hypertensive children with renal disease. $\mathcal{f}$ Pediatr 1983;103:799-805.

12 Schneeweiss A. Cardiovascular drugs in children. II. Angiotensin-converting enzyme inhibitors in Pediatric patients. Pediatr Cardiol 1990;11:199-207.

13 Echigo S. PTCA. In: Abstracts of the third International Kawasaki Disease Symposium. Tokyo 1988;64.

14 Ino $T$, Nishimoto $K$, Akimoto $K$, Park I, Shimazaki $S$, Yabuta $\mathrm{K}$, et al. Percutaneous transluminal coronary angioplasty for Kawasaki disease: A case report and literature review. Pediatr Cardiol 1991;12:33-5.

\section{IMAGES IN CARDIOLOGY}

\section{Damage to an internal mammary artery graft at dissection}

The internal mammary artery makes an excellent coronary graft with the potential for long-term patency and consequent relief of angina, freedom from infarction, and an improved chance of survival. But dissecting it and sewing it on is not enough-it has to provide blood flow. One hears loose talk about "spasm" and unsubstantiated remarks about its cause and many ways of relieving it. But canny surgeons like to see a brisk flow of blood come out the end of the graft before committing the future of the LAD territory to what is, at the time of operation, often an insubstantial little artery. An internal mammary artery that was not accepted on this basis is shown here, cut open longitudinally. A circumferential intimal flap was revealedthe result presumably of inadvertent traction during dissection by an inexperienced operator. This pathological entity is well recognised in limb arteries where it is due to abrupt traction at the time of injury. The arterial pulse does not return after the fracture has been reduced and an intimal flap, with overlying thrombus, is found on exploration of the artery. The same lesion can occur in the internal mammary artery, as shown here, and would be a more potent cause of postoperative ischaemia than "spasm".

TOM TREASURE

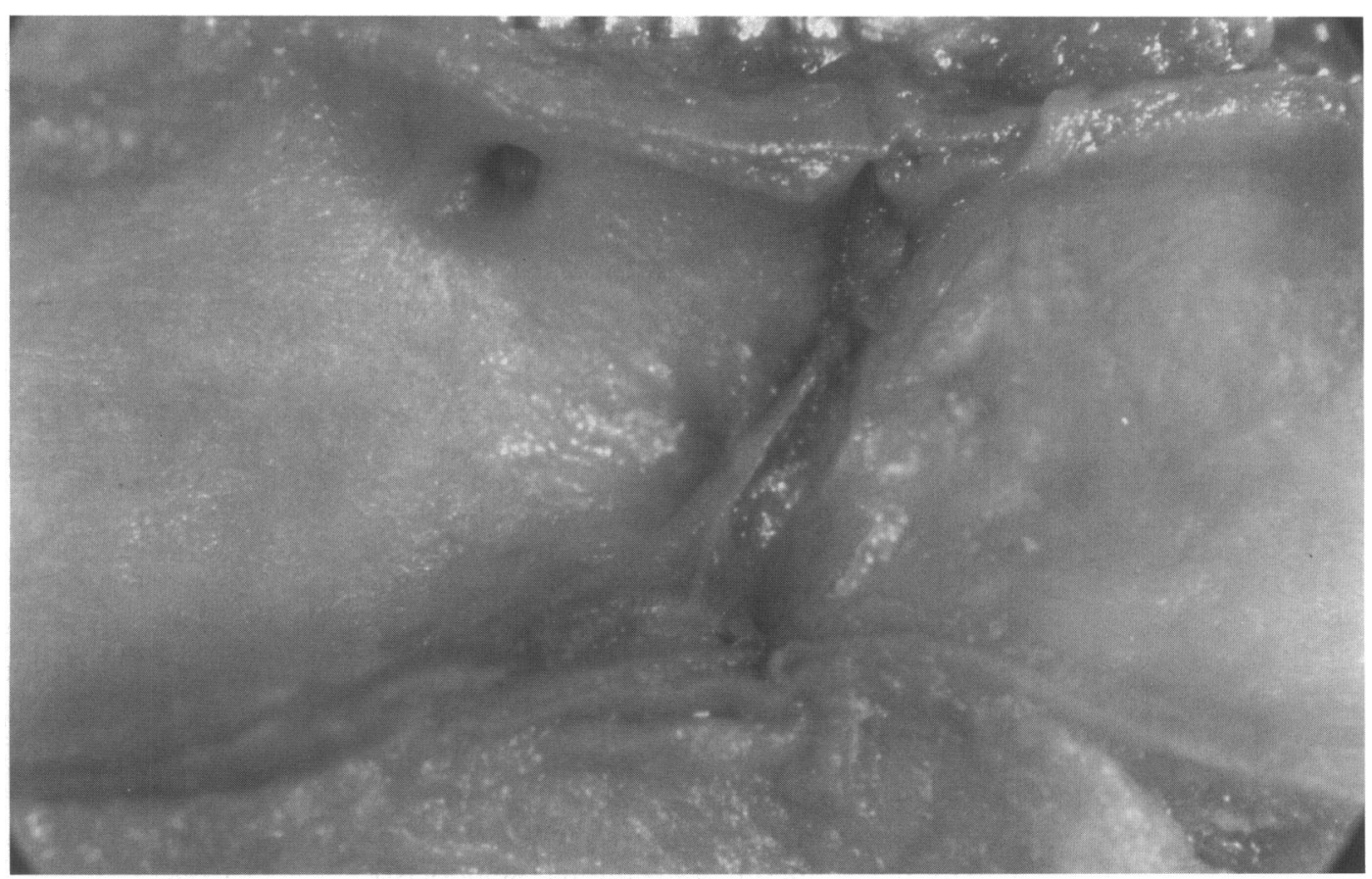

